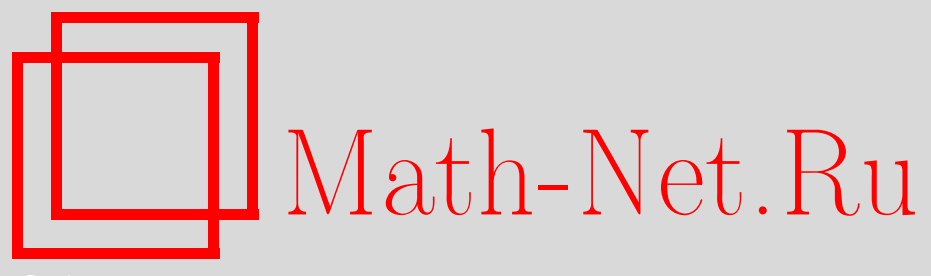

А. В. Устинов, О среднем числе шагов в алгоритме Евклида с нечетными неполными частными, Матем. заметки, 2010, том 88, выпуск 4, 594-604

DOI: https://doi.org/10.4213/mzm8854

Использование Общероссийского математического портала Math-Net.Ru подразумевает, что вы прочитали и согласны с пользовательским соглашением http://www . mathnet.ru/rus/agreement

Параметры загрузки:

IP: 54.162 .27 .143

26 апреля 2023 г., 13:45:41

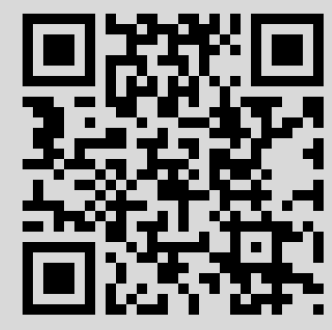


Том 88 выпуск 4 октябрь 2010

УДК $517.524+510.52+519.712 .61$

\section{О среднем числе шагов в алгоритме Евклида \\ с нечетными неполными частными}

\section{А. В. Устинов}

Длина разложения рационального числа в цепную дробь с нечетными неполными частными выражается через статистики Гаусса-Кузьмина для классической цепной дроби. Благодаря этому для средней длины алгоритма Евклида с нечетными неполными частными удается доказать асимптотические формулы аналогичные тем, которые ранее были известны для классического алгоритма Евклида.

Библиография: 12 названий.

Памлти Анатолия Алексеевича Карачубъ

1. Три быстрых варианта алгоритма Евклида и статистики Гаусса-Кузьмина. Среди различных модификаций алгоритма Евклида обычно выделяют три "быстрых" варианта (см. [1]), основанных на различных способах деления с остатком:

- классический алгоритм Евклида

$$
a=b q+r, \quad q=\left\lfloor\frac{a}{b}\right\rfloor, \quad 0 \leqslant r<b ;
$$

- алгоритм с выбором минимального по модулю остатка

$$
a=b q+\varepsilon r, \quad \varepsilon= \pm 1, \quad q=\left\lceil\frac{a}{b}-\frac{1}{2}\right\rceil, \quad-\frac{b}{2}<r \leqslant \frac{b}{2} ;
$$

- алгоритм с нечетными неполными частными

$$
a=b q+\varepsilon r, \quad \varepsilon= \pm 1, \quad q=2\left\lceil\frac{a}{2 b}\right\rceil-1, \quad-b<r \leqslant b .
$$

Число шагов в быстрых вариантах в наихудшем случае и в среднем есть $O(\log N)$ (при $a, b \ll N)$.

K "медленным" относят: алгоритм с вычитанием, алгоритм с делением "по избытку" и алгоритм с четными неполными частными. Для них число шагов в среднем

Работа выполнена при поддержке гранта Президента РФ № МД-2339.2010.1, фонда “Династия", Российского фонда фундаментальных исследований (гранты №o 09-01-12129-офи-м, 10-01-98001-р-сибирь-а, 09-01-00371-а) и проекта ДВО № 09-I-П4-03.

(C) А.В. Устинов, 2010 
имеет порядок $\log ^{2} N$, а в наихудших случаях может быть порядка $N$ (более подробная информация и библиография могут быть найдены в [1]).

Каждый из трех быстрых алгоритмов приводит к разложению чисел в соотвествующие цепные дроби. Классический алгоритм:

$$
\frac{a}{b}=a_{0}+\frac{1}{a_{1}+\cdot+\frac{1}{a_{s}}}=\left[a_{0} ; a_{1}, \ldots, a_{s}\right]
$$

где $a_{0}$ - целое, $a_{1}, \ldots, a_{s}$ - натуральные и $a_{s} \geqslant 2$ при $s \geqslant 1$. (Другой вариант обеспечить однозначность разложения - это требовать, чтобы последнее неполное частное было равно единице.) Алгоритм с выбором минимального по модулю остатка:

$$
\frac{a}{b}=a_{0}+\frac{\varepsilon_{1}}{a_{1}+\frac{\varepsilon_{2}}{a_{2}+\cdot \cdot+\frac{\varepsilon_{l}}{a_{l}}}},
$$

где $a_{0}$ - целое, $a_{1}, \ldots, a_{l}$ - натуральные, $\varepsilon_{k}= \pm 1, a_{k} \geqslant 2,1 \leqslant k \leqslant l, a_{k}+\varepsilon_{k+1} \geqslant 2$, $1 \leqslant k<l$, и $\varepsilon_{l}=-1$ при $l \geqslant 1$ и $a_{l}=2$. Алгоритм с нечетными неполными частными:

$$
\frac{a}{b}=a_{0}+\frac{\varepsilon_{1}}{a_{1}+\frac{\varepsilon_{2}}{a_{2}+\ddots \cdot+\frac{\varepsilon_{h}}{a_{h}}}}=\left\langle a_{0} ; \frac{\varepsilon_{1}}{a_{1}}, \frac{\varepsilon_{2}}{a_{2}}, \ldots, \frac{\varepsilon_{h}}{a_{h}}\right\rangle,
$$

где $a_{0}$ - нечетное целое, $a_{1}, \ldots, a_{h}$ - нечетные натуральные, $\varepsilon_{k}= \pm 1, a_{k}+\varepsilon_{k+1} \geqslant 1$, $1 \leqslant k<h$, и $\varepsilon_{h}=1$ при $h \geqslant 1$ и $a_{h}=1$. Длины разложений рационального числа $a / b$ в дроби вида (1)-(3) будем соответственно обозначать $s(a / b), l(a / b)$ и $h(a / b)$.

$\mathrm{C}$ вычислительной точки зрения естественно возникает задача об исследовании средних значений функций $s(a / b), l(a / b)$ и $h(a / b)$. Вопрос о средней длине классических цепных дробей впервые был исследован Хейльбронном. В 1968 г. элементарными методами он доказал асимптотическую формулу (см. [2])

$$
\frac{1}{\varphi(b)} \sum_{a=1}^{b} s\left(\frac{a}{b}\right)=\frac{2 \log 2}{\zeta(2)} \cdot \log b+O\left(\log ^{4} \log b\right)
$$

(здесь и далее знак звездочки в суммах означает, что переменная суммирования пробегает приведенную систему вычетов). В 1975 г. Портер, используя оценки сумм Клостермана, для того же среднего получил асимптотическую формулу с двумя значащими членами (см. [3])

$$
\frac{1}{\varphi(b)} \sum_{a=1}^{b} s\left(\frac{a}{b}\right)=\frac{2 \log 2}{\zeta(2)} \cdot \log b+C_{P}-1+O_{\varepsilon}\left(b^{-1 / 6+\varepsilon}\right),
$$

где $\varepsilon-$ любое положительное и

$$
C_{P}=\frac{2 \log 2}{\zeta(2)}\left(\frac{3 \log 2}{2}+2 \gamma-2 \frac{\zeta^{\prime}(2)}{\zeta(2)}-1\right)-\frac{1}{2}
$$


- константа, получившая название константы Портера (ее окончательный вид был найден Ренчем, см. [4]). При усреднении по обоим параметрам можно доказать более точную формулу (см. [5])

$$
\frac{2}{R(R+1)} \sum_{b \leqslant R} \sum_{a=1}^{b} s\left(\frac{a}{b}\right)=\frac{2 \log 2}{\zeta(2)} \cdot \log R+\widetilde{C}_{P}-1+O\left(R^{-1} \log R^{4}\right),
$$

где

$$
\widetilde{C}_{P}=C_{P}+\frac{2 \log 2}{\zeta(2)}\left(\frac{\zeta^{\prime}(2)}{\zeta(2)}-\frac{1}{2}\right)
$$

Для цепных дробей вида (2) и (3) аналоги результата Хейльбронна были доказаны Ригером (см. [6], [7]). В работе [8] Балади и Валле эргодическими методами доказали двучленные асимптотические формулы

$$
\begin{aligned}
& \frac{2}{R(R+1)} \sum_{b \leqslant R} \sum_{a=1}^{b} l\left(\frac{a}{b}\right)=\frac{2 \log \varphi}{\zeta(2)} \cdot \log R+\widetilde{C}_{l}+O\left(R^{-\gamma}\right), \\
& \frac{2}{R(R+1)} \sum_{b \leqslant R} \sum_{a=1}^{b} h\left(\frac{a}{b}\right)=\frac{3 \log \varphi}{\zeta(2)} \cdot \log R+\widetilde{C}_{h}+O\left(R^{-\gamma}\right),
\end{aligned}
$$

где $\varphi=(1+\sqrt{5}) / 2$ - "золотое сечение" и $\gamma>0$. В той же работе был поставлен вопрос о нахождении констант $\widetilde{C}_{l}$ и $\widetilde{C}_{h}$.

Оказывается, что вопрос о поведении в среднем функций $l(a / b)$ и $h(a / b)$ можно свести к анализу классического алгоритма Евклида, если воспользоваться статистиками Гаусса-Кузьмина. Это позволяет для средних значений $l(a / b)$ и $h(a / b)$ доказать формулы, аналогичные $(4)$ и $(5)$, а для констант $\widetilde{C}_{l}$ и $\widetilde{C}_{h}$ получить представления через сингулярные ряды.

Для рационального $a / b=\left[0 ; a_{1}, \ldots, a_{s}, 1\right] \in[0,1]$ и действительных $x \in[0,1] c m a$ тистиками Гаусса-Кузъмина называются числа (см. [9])

$$
s_{x}\left(\frac{a}{b}\right)=\#\left\{j: 0 \leqslant j \leqslant s,\left[0 ; a_{j+1}, \ldots, a_{s}, 1\right] \leqslant x\right\} .
$$

В частности, $s_{1}(a / b)=s+1$ - длина работы классического алгоритма Евклида, примененного к паре чисел $(a, b)$ (при этом подразумевается, что на первом шаге переменные переставляются, а на следующих шагах уже происходят деления с остатком, см. [10]).

Для дальнейших рассуждений оказывается удобным распространить определение статистик Гаусса-Кузьмина на произвольные $x>0$ :

$$
s_{x}\left(\frac{a}{b}\right)=\#\left\{(j, t): 0 \leqslant j \leqslant s, 0 \leqslant t<a_{j},\left[t ; a_{j+1}, \ldots, a_{s}, 1\right] \leqslant x\right\}
$$

(здесь считаем, что $\left.a_{0}=+\infty\right)$. Для конечных цепных дробей выбрана запись с единицей на конце, чтобы новое определение (7) совпадало со старым (6) не только при $x \in[0,1)$, но и при $x=1$. 
Для средних значений статистик Гаусса-Кузьмина выполняются равенства (доказательства из работ [5], [11] остаются справедливыми при произвольном положительном $x$ )

$$
\begin{aligned}
\frac{1}{\varphi(b)} \sum_{a=1}^{b} s_{x}\left(\frac{a}{b}\right) & =\frac{2 \log (1+x)}{\zeta(2)} \log b+C_{P}(x)+O\left(b^{-1 / 6} \log ^{7 / 6+\varepsilon} b\right), \\
\frac{2}{R(R+1)} \sum_{b \leqslant R} \sum_{a=1}^{b} s_{x}\left(\frac{a}{b}\right) & =\frac{2 \log (1+x)}{\zeta(2)} \log R+\widetilde{C}_{P}(x)+O\left(R^{-1} \log ^{4} R\right),
\end{aligned}
$$

где

$$
\begin{aligned}
C_{P}(x)= & \frac{2 \log (1+x)}{\zeta(2)}\left(2 \gamma-2 \frac{\zeta^{\prime}(2)}{\zeta(2)}-\frac{\log (1+x)}{2}+\log x-1\right) \\
& +\frac{2}{\zeta(2)}\left(h_{1}(x)+h_{2}(x)\right)+\frac{x^{2}}{x+1}, \\
\widetilde{C}_{P}(x)= & C_{P}(x)+\frac{2 \log (1+x)}{\zeta(2)}\left(\frac{\zeta^{\prime}(2)}{\zeta(2)}-\frac{1}{2}\right),
\end{aligned}
$$

а функции $h_{1}(x)$ и $h_{2}(x)$ задаются абсолютно сходящимися сингулярными рядами

$$
\begin{aligned}
& h_{1}(x)=\sum_{n=1}^{\infty} \frac{1}{n}\left(\sum_{m=1}^{n} \frac{x}{n+m x}-\log (1+x)\right), \\
& h_{2}(x)=\sum_{n=1}^{\infty} \frac{1}{n}\left(\sum_{n / x \leqslant m<n / x+n} \frac{1}{m}-\log (1+x)\right) .
\end{aligned}
$$

В статье [12] доказано, что $l(a / b)=s_{\varphi-1}(a / b)$. Поэтому результаты о средних значениях $l(a / b)$ (и формулы для вторых констант в асимптотических формулах) получаются подстановкой $x=\varphi-1$ в формулы (8) и (9).

В настоящей статье доказывается, что при $b \geqslant 1, b / 2 \leqslant a<b,(a, b)=1, a a^{\star} \equiv 1$ $(\bmod b), 1 \leqslant a^{\star}<b$ выполняется тождество

$$
h\left(\frac{a^{\star}}{b}\right)+h\left(\frac{b-a^{\star}}{b}\right)=s_{\varphi}\left(\frac{a}{b}\right)+s_{\varphi-1}\left(\frac{a}{b}\right) .
$$

Поэтому равенства (8) и (9) приводят к следующему результату.

Теорема. Справедливы асимптотические формуль

$$
\begin{aligned}
\frac{1}{\varphi(b)} \sum_{a=1}^{b} h\left(\frac{a}{b}\right) & =\frac{3 \log \varphi}{\zeta(2)} \log b+C_{h}+O\left(b^{-1 / 6} \log ^{7 / 6+\varepsilon} b\right), & b \geqslant 2, \\
\frac{2}{R(R+1)} \sum_{b \leqslant R} \sum_{a=1}^{b} h\left(\frac{a}{b}\right) & =\frac{3 \log \varphi}{\zeta(2)} \log R+\widetilde{C}_{h}+O\left(R^{-1} \log ^{4} R\right), & R \geqslant 2,
\end{aligned}
$$

где

$$
\begin{aligned}
C_{h} & =\frac{1}{2}\left(C_{P}(\varphi)+C_{P}(\varphi-1)\right), \\
\widetilde{C}_{h} & =\frac{1}{2}\left(\widetilde{C}_{P}(\varphi)+\widetilde{C}_{P}(\varphi-1)\right)=C_{h}+\frac{3 \log \varphi}{\zeta(2)}\left(\frac{\zeta^{\prime}(2)}{\zeta(2)}-\frac{1}{2}\right),
\end{aligned}
$$

и функиии $C_{P}(x), \widetilde{C}_{P}(x)$ определены равенствами (10), (11). 
2. Разложения в дуальные дроби. Наряду с разложениями вида (3) будем рассматривать дуальные (перевернутые) дроби

$$
\left\langle 0 ; \frac{\varepsilon_{h+1}}{a_{h}}, \ldots, \frac{\varepsilon_{2}}{a_{1}}\right\rangle^{\star}=\frac{\varepsilon_{h+1}}{a_{h}+\cdot \cdot+\frac{\varepsilon_{2}}{a_{1}}}
$$

с теми же ограничениями на параметры: $a_{j}$ - нечетные натуральные числа, $1 \leqslant$ $j \leqslant h, \varepsilon_{j}= \pm 1,2 \leqslant j \leqslant h+1, \varepsilon_{j+1}+a_{j}>0,1 \leqslant j<h$.

Лемма 1. Рациональное число $p / q$ представимо дуальной дробъю тогда и только тогда, когда $p / q \in(\varphi-2, \varphi)$; представление дуалъной дробъю единственно.

ДокАЗАТЕЛЬСтво. Необходимость условия $p / q \in(\varphi-2, \varphi)$ следует из неравенств

$$
\varphi-2=\left\langle 0 ; \frac{-1}{3}, \frac{-1}{3}, \ldots\right\rangle^{\star}<\left\langle 0 ; \frac{\varepsilon_{h+1}}{a_{h}}, \ldots, \frac{\varepsilon_{2}}{a_{1}}\right\rangle^{\star}<\left\langle 0 ; \frac{1}{1}, \frac{-1}{3}, \frac{-1}{3}, \ldots\right\rangle^{\star}=\varphi .
$$

Для доказательства его достаточности опишем алгоритм разложения. Имеем при $\varepsilon_{h+1}=-1$

$$
-\frac{1}{\varphi^{2}}=\varphi-2<\frac{p}{q}<0, \quad-\frac{q}{p}>\varphi^{2}
$$

и для некоторого нечетного $a_{h}>\varphi^{2}-\varphi=1$, определяемого однозначно, $-a_{h}-q / p$ будет лежать в интервале $(\varphi-2, \varphi)$. Если же $\varepsilon_{h+1}=1$, то $q / p>1 / \varphi$ и для некоторого нечетного

$$
a_{h} \geqslant \frac{1}{\varphi}+\frac{1}{\varphi^{2}}=1,
$$

также определяемого однозначно, $q / p-a_{h}$ также попадет в интервал $(\varphi-2, \varphi)$. Таким образом, в каждом из случаев однозначно находится пара $\left(\varepsilon_{h+1}, a_{h}\right)$, для которой $\varepsilon_{h+1}+a_{h}>0$ и осуществляется переход к дроби

$$
\frac{r}{p}= \pm \frac{q}{p}-a_{h} \in(\varphi-2, \varphi) .
$$

Разложение будет конечным, поскольку на каждом шаге уменьшается высота раскладываемого числа $(|r|+|p|<|p|+q)$. Единственность представления дуальной дробью следует из однозначности описанного алгоритма.

Длину разложения рационального числа $a / b$ в дуальную дробь будем далее обозначать через $h^{\star}(a / b)$.

3. Цепные дроби и матрицы. При изучении статистических свойств конечных цепных дробей ключевую роль (см., например, [5], [11]) играет биекция, которая каждому непустому набору натуральных чисел $\left(a_{1}, \ldots, a_{n}\right)$ (составленному из неполных частных рационального числа) ставит в соответствие матрицу

$$
\left(\begin{array}{cc}
0 & 1 \\
1 & a_{1}
\end{array}\right) \cdots\left(\begin{array}{cc}
0 & 1 \\
1 & a_{s}
\end{array}\right) \in \mathscr{M}
$$

где

$$
\mathscr{M}=\left\{\left(\begin{array}{ll}
P & P^{\prime} \\
Q & Q^{\prime}
\end{array}\right) \in \mathrm{GL}_{2}(\mathbb{Z}): P \geqslant 0,1 \leqslant P^{\prime} \leqslant Q^{\prime}, 1 \leqslant Q \leqslant Q^{\prime}\right\} .
$$


Для изучения цепных дробей с нечетными неполными частными и дуальных к ним дробей введем отображение $\Phi$, которое будет ставить в соответствие рациональному числу

$$
\frac{a}{b}=\left\langle 0 ; \frac{\varepsilon_{h+1}}{a_{h}}, \ldots, \frac{\varepsilon_{2}}{a_{1}}\right\rangle^{\star} \in(\varphi-2, \varphi)
$$

матрицу

$$
\Phi\left(\frac{a}{b}\right)=\left(\begin{array}{cc}
0 & \varepsilon_{h+1} \\
1 & a_{h}
\end{array}\right) \cdots\left(\begin{array}{cc}
0 & \varepsilon_{2} \\
1 & a_{1}
\end{array}\right) .
$$

Отметим основные свойства отображения $\Phi$.

$1^{\circ}$. Если $\Phi(a / b)=\left(\begin{array}{cc}P & P^{\prime} \\ Q & Q^{\prime}\end{array}\right)$, то

$$
\begin{aligned}
\frac{P}{Q} & =\left\langle 0 ; \frac{\varepsilon_{h+1}}{a_{h}}, \ldots, \frac{\varepsilon_{3}}{a_{2}}\right\rangle^{\star}, & \frac{P}{P^{\prime}} & =\left\langle 0 ; \frac{1}{a_{1}}, \frac{\varepsilon_{2}}{a_{2}}, \ldots, \frac{\varepsilon_{h-1}}{a_{h-1}}\right\rangle, \\
\frac{P^{\prime}}{Q^{\prime}} & =\left\langle 0 ; \frac{\varepsilon_{h+1}}{a_{h}}, \ldots, \frac{\varepsilon_{2}}{a_{1}}\right\rangle^{\star}=\frac{a}{b}, & \frac{Q}{Q^{\prime}} & =\left\langle 0 ; \frac{1}{a_{1}}, \frac{\varepsilon_{2}}{a_{2}}, \ldots, \frac{\varepsilon_{h}}{a_{h}}\right\rangle .
\end{aligned}
$$

$2^{\circ}$. Всякая матрица $S=\left(\begin{array}{ll}P & P^{\prime} \\ Q & Q^{\prime}\end{array}\right)$, лежащая в образе отображения $\Phi$ однозначно восстанавливается как по строке $\left(Q Q^{\prime}\right)$, так и по столбцу $\left(\begin{array}{l}P^{\prime} \\ Q^{\prime}\end{array}\right)$. В частности, определитель матрицы $S$ есть функция от отношения $P^{\prime} / Q^{\prime}$, которую в дальнейшем будем обозначать через $\Delta\left(P^{\prime} / Q^{\prime}\right)$.

$3^{\circ}$. Отображение $\Phi$ является биекцией между $\mathbb{Q} \cap(\varphi-2, \varphi)$ и множеством матриц

$$
\mathscr{N}=\left\{\left(\begin{array}{ll}
P & P^{\prime} \\
Q & Q^{\prime}
\end{array}\right) \in \mathrm{GL}_{2}(\mathbb{Z}): 1 \leqslant Q \leqslant Q^{\prime}, P Q^{\prime}-P^{\prime} Q=\Delta\left(\frac{P^{\prime}}{Q^{\prime}}\right), \frac{P^{\prime}}{Q^{\prime}} \in(\varphi-2, \varphi)\right\} .
$$

$4^{\circ}$. Если $\left(\begin{array}{ll}P & P^{\prime} \\ Q & Q^{\prime}\end{array}\right) \in \mathscr{N}$, то $h^{\star}\left(P^{\prime} / Q^{\prime}\right)=h\left(Q / Q^{\prime}\right)$.

$5^{\circ}$. Если $Q^{\prime} \geqslant 2$ и

$$
\Phi\left(\frac{a}{b}\right)=\left(\begin{array}{ll}
* & P^{\prime} \\
Q & Q^{\prime}
\end{array}\right) \in \mathscr{M} \cap \mathscr{N}
$$

то $\Delta\left(\left(Q^{\prime}-P^{\prime}\right) / Q^{\prime}\right)=\Delta\left(P^{\prime} / Q^{\prime}\right)$ и

$$
\Phi\left(\frac{b-a}{b}\right)=\left(\begin{array}{cc}
* & Q^{\prime}-P^{\prime} \\
Q^{\prime}-Q & Q^{\prime}
\end{array}\right) \in \mathscr{M} \cap \mathscr{N} .
$$

$6^{\circ}$. При $Q^{\prime} \geqslant 2$ строка $\left(Q Q^{\prime}\right)$ и столбец $\left(\begin{array}{l}P^{\prime} \\ Q^{\prime}\end{array}\right)$ могут быть двумя способами дополнены до матрицы из $\mathscr{M}$. При этом в каждой из двух пар полученных матриц

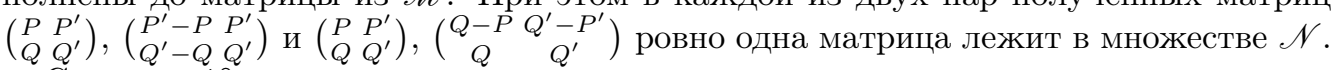

Свойство $1^{\circ}$ проверяется по индукции.

Свойство $2^{\circ}$ непосредственно следует из свойства $1^{\circ}$.

В свойстве $3^{\circ}$ инъективность отображения $\Phi$ следует из леммы 1 и равенства $P^{\prime} / Q^{\prime}=a / b$. Для доказательства сюръективности рассмотрим произвольную мат-

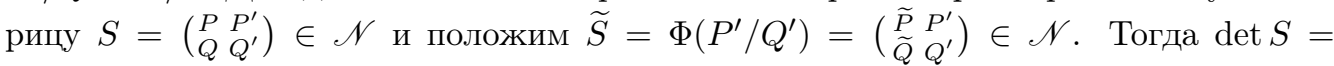
$\operatorname{det} \widetilde{S}=\Delta\left(P^{\prime} / Q^{\prime}\right)$, и для некоторого целого $t$ выполняются равенства $\widetilde{P}=P+t P^{\prime}$, $\widetilde{Q}=Q+t Q^{\prime}$. Но $1 \leqslant Q, \widetilde{Q} \leqslant Q^{\prime}$, следовательно, $t=0$ и $S=\widetilde{S}$.

Свойство $4^{\circ}$ вытекает из свойства $3^{\circ}$ и равенств (13). 
Для доказательства свойства $5^{\circ}$ сначала заметим, что при $Q^{\prime}=2$ есть ровно одна матрица $S=\left(\begin{array}{ll}1 & 1 \\ 1 & 2\end{array}\right)$, лежащая в $\mathscr{M} \cap \mathscr{N}$. Для нее утверждение леммы очевидно, поэтому можно считать, что $Q^{\prime} \geqslant 3$. Согласно свойству $2^{\circ}$, столбец $\left(\begin{array}{c}Q^{\prime}-P^{\prime} \\ Q^{\prime}\end{array}\right)$ можно дополнить до некоторой матрицы $\widetilde{S}=\left(\begin{array}{c}* Q^{\prime}-P^{\prime} \\ Q^{\prime}\end{array}\right) \in \mathscr{N}$. По определению (12) эта матрица лежит в множестве $\mathscr{M}$ и $1 \leqslant \widetilde{Q}<Q^{\prime}$. При этом $\widetilde{Q} \neq Q$, так как $Q^{\prime}-P^{\prime} \neq P^{\prime}$. Поскольку $\operatorname{det} S= \pm \operatorname{det} \widetilde{S}$, то $Q P^{\prime} \equiv \pm \widetilde{Q} P^{\prime}\left(\bmod Q^{\prime}\right)$, и $\widetilde{Q}=Q^{\prime}-Q$. Значит, имеем $\operatorname{det} S \equiv P^{\prime} Q \equiv \operatorname{det} \widetilde{S}\left(\bmod Q^{\prime}\right)$ и $\Delta\left(\left(Q^{\prime}-P^{\prime}\right) / Q^{\prime}\right)=\Delta\left(P^{\prime} / Q^{\prime}\right)$.

Свойство $6^{\circ}$ следует из свойства $2^{\circ}$.

\section{4. Сведение к статистикам Гаусса-Кузьмина.}

Лемма 2. Пусть $b \geqslant 2, b / 2 \leqslant a<b,(a, b)=1$. Тогдa

$$
h^{\star}\left(\frac{a}{b}\right)+h^{\star}\left(\frac{b-a}{b}\right)=s_{\varphi}\left(\frac{a}{b}\right)+s_{\varphi-1}\left(\frac{a}{b}\right) .
$$

ДокАЗАтЕЛьство. Пусть $a / b=\left[0 ; 1, a_{1}, \ldots, a_{s}, 1\right](s \geqslant 0)$. Каждому $k$ в пределах $0 \leqslant k \leqslant s$ будем ставить в соответствие четверку чисел $\left(u_{k}, v_{k}, p_{k}, q_{k}\right)$, определяемую равенствами $\left(u_{k}, v_{k}\right)=\left(p_{k}, q_{k}\right)=1$,

$$
\frac{u_{k}}{v_{k}}=\left[0 ; a_{k}, \ldots, a_{1}, 1\right], \quad \frac{p_{k}}{q_{k}}=\left[0 ; a_{k+1}, \ldots, a_{s}, 1\right] .
$$

Определим мультимножество (его элементы могут повторяться) $A=A_{1} \cup A_{2} \cup A_{3}$, где

$$
\begin{aligned}
& A_{1}=\left\{\left(u_{k}, v_{k}, p_{k}, q_{k}\right): 0 \leqslant k \leqslant s\right\}, \\
& A_{2}=\left\{\left(u_{k}, v_{k}, p_{k}, q_{k}\right): 0 \leqslant k \leqslant s, u_{k} \leqslant \frac{v_{k}}{2}, \frac{p_{k}}{q_{k}}<\frac{1}{\varphi}\right\}, \\
& A_{3}=\left\{\left(u_{k}, v_{k}, p_{k}, q_{k}\right): 0 \leqslant k \leqslant s, v_{k} \geqslant 2, \frac{p_{k}}{q_{k}}<\frac{1}{\varphi}\right\} .
\end{aligned}
$$

При этом кратность вхождения каждой четверки в множество $A$ равна сумме кратностей, с которыми эта четверка входит в $A_{1}, A_{2}$ и $A_{3}$.

Разложению

$$
\frac{a}{b}=\left\langle 0 ; \frac{1}{b_{h}}, \frac{\varepsilon_{h}}{b_{h-1}}, \ldots, \frac{\varepsilon_{2}}{b_{1}}\right\rangle^{\star}
$$

и номеру $j$ в пределах $2 \leqslant j \leqslant h$ будем ставить в соответствие четверку чисел $\left(\alpha_{j}, \beta_{j}, \lambda_{j}, \mu_{j}\right)$, определяемую условиями $\alpha_{j}, \beta_{j}, \mu_{j}>0,\left(\alpha_{j}, \beta_{j}\right)=\left(\lambda_{j}, \beta_{j}\right)=1$,

$$
\frac{\alpha_{j}}{\beta_{j}}=\left\langle 0 ; \frac{1}{b_{j}}, \frac{\varepsilon_{j+1}}{b_{j+1}}, \ldots, \frac{\varepsilon_{h}}{b_{h}}\right\rangle, \quad \frac{\lambda_{j}}{\mu_{j}}=\left\langle 0 ; \frac{\varepsilon_{j}}{b_{j-1}}, \ldots, \frac{\varepsilon_{2}}{b_{1}}\right\rangle^{\star} .
$$

Через $B$ обозначим множество всех таких четверок:

$$
B=\left\{\left(\alpha_{j}, \beta_{j}, \lambda_{j}, \mu_{j}\right): 2 \leqslant j \leqslant h\right\} .
$$


При этом $B=B_{1} \cup B_{2} \cup B_{3}$, где

$$
\begin{aligned}
& B_{1}=\left\{(\alpha, \beta, \lambda, \mu) \in B: 0<\frac{\lambda}{\mu} \leqslant 1\right\}, \\
& B_{2}=\left\{(\alpha, \beta, \lambda, \mu) \in B: 1<\frac{\lambda}{\mu}<\varphi\right\}, \\
& B_{3}=\left\{(\alpha, \beta, \lambda, \mu) \in B: \varphi-2<\frac{\lambda}{\mu}<0\right\} .
\end{aligned}
$$

Через $\widetilde{B}, \widetilde{B}_{1}, \widetilde{B}_{2}$ и $\widetilde{B}_{3}$ обозначим аналогичные множества, построенные из разложения $(b-a) / b$ в дуальную дробь.

По определению статистик Гаусса-Кузьмина

$$
\#\left(A_{1} \cup A_{2}\right)=s_{\varphi}\left(\frac{a}{b}\right)-1, \quad \# A_{3}=s_{\varphi-1}\left(\frac{a}{b}\right)-1 .
$$

Кроме того,

$$
\# B=h^{\star}\left(\frac{a}{b}\right)-1, \quad \# \widetilde{B}=h^{\star}\left(\frac{b-a}{b}\right)-1 .
$$

Поэтому для доказательства леммы достаточно проверить равномощность множеств $A$ и $B \cup \widetilde{B}$. Для этого укажем отображения $f_{i}, i=1,2,3$, которые устанавливают взаимно однозначные отображения из $A_{i}$ в $B_{i} \cup \widetilde{B}_{i}$ :

$$
\begin{aligned}
& f_{1}(u, v, p, q)=(u, v, p, q), \\
& f_{2}(u, v, p, q)=(u, v-u, p+q, q), \\
& f_{3}(u, v, p, q)=(v-u, v,-p, p+q) .
\end{aligned}
$$

Например, если $(u, v, p, q) \in A_{1}$, то для некоторых $u^{\prime}$ и $v^{\prime}$

$$
\left(\begin{array}{ll}
u^{\prime} & v^{\prime} \\
u & v
\end{array}\right)\left(\begin{array}{ll}
* & p \\
* & q
\end{array}\right)=\left(\begin{array}{ll}
* & a \\
* & b
\end{array}\right)
$$

где каждый из сомножителей лежит в $\mathscr{M}$. Если при этом $\left(\begin{array}{c}u^{\prime} \\ u \\ v\end{array}\right) \in \mathscr{N}$, то $(u, v, p, q) \in$ $B_{1}$, если же $\left(\begin{array}{cc}u^{\prime} & v^{\prime} \\ u & v\end{array}\right) \notin \mathscr{N}$, то $\left(\begin{array}{cc}u-u^{\prime} & v-v^{\prime} \\ u & v\end{array}\right) \in \mathscr{N}\left(\right.$ см. свойство $\left.6^{\circ}\right)$,

$$
\left(\begin{array}{cc}
u-u^{\prime} & v-v^{\prime} \\
u & v
\end{array}\right)\left(\begin{array}{cc}
* & p \\
* & q
\end{array}\right)=\left(\begin{array}{cc}
* & b-a \\
* & b
\end{array}\right),
$$

и $(u, v, p, q) \in \widetilde{B}_{1}$. При этом каждая четверка $(\alpha, \beta, \lambda, \mu) \in B_{1} \cup \widetilde{B}_{1}$ будет лежать в образе $f_{1}$. Так, если $(\alpha, \beta, \lambda, \mu) \in B_{1}$, то (сомножители лежат в $\left.\mathscr{N}\right)$

$$
\left(\begin{array}{cc}
\alpha^{\prime} & \beta^{\prime} \\
\alpha & \beta
\end{array}\right)\left(\begin{array}{ll}
* & \lambda \\
* & \mu
\end{array}\right)=\left(\begin{array}{ll}
* & a \\
* & b
\end{array}\right) .
$$

Первый сомножитель лежит и в множестве $\mathscr{M}$, а матрицы $\left(\begin{array}{ll}* & \lambda \\ * & \mu\end{array}\right)$ и $\left(\begin{array}{ll}* & a \\ * & b\end{array}\right)$ можно превратить в матрицы из $\mathscr{M}$, изменив первые столбцы (см. свойство $6^{\circ}$ ). Значит, $(\alpha, \beta, \lambda, \mu) \in A_{1}$. 
Если же $(\alpha, \beta, \lambda, \mu) \in \widetilde{B}_{1}$, то

$$
\left(\begin{array}{cc}
\alpha^{\prime} & \beta^{\prime} \\
\alpha & \beta
\end{array}\right)\left(\begin{array}{cc}
* & \lambda \\
* & \mu
\end{array}\right)=\left(\begin{array}{cc}
* & b-a \\
* & b
\end{array}\right), \quad\left(\begin{array}{cc}
\alpha-\alpha^{\prime} & \beta-\beta^{\prime} \\
\alpha & \beta
\end{array}\right)\left(\begin{array}{ll}
* & \lambda \\
* & \mu
\end{array}\right)=\left(\begin{array}{ll}
* & a \\
* & b
\end{array}\right) .
$$

В последнем равенстве каждый из сомножителей (быть может, после замены элементов, помеченных звездочками) будет лежать в $\mathscr{M}$, а значит, и в этом случае $(\alpha, \beta, \lambda, \mu) \in A_{1}$.

Биективность $f_{2}$ и $f_{3}$ проверяется аналогично, исходя из равенств

$$
\begin{aligned}
\left(\begin{array}{ll}
u^{\prime} & v^{\prime} \\
u & v
\end{array}\right)\left(\begin{array}{ll}
p^{\prime} & p \\
q^{\prime} & q
\end{array}\right) & =\left(\begin{array}{cc}
u^{\prime} & v^{\prime}-u^{\prime} \\
u & v-u
\end{array}\right)\left(\begin{array}{cc}
p^{\prime}+q^{\prime} & p+q \\
q^{\prime} & q
\end{array}\right), \\
\left(\begin{array}{ll}
u^{\prime} & v^{\prime} \\
u & v
\end{array}\right)\left(\begin{array}{ll}
p^{\prime} & p \\
q^{\prime} & q
\end{array}\right) & =\left(\begin{array}{cc}
v^{\prime}-u^{\prime} & v^{\prime} \\
v-u & v
\end{array}\right)\left(\begin{array}{cc}
-p^{\prime} & -p \\
p^{\prime}+q^{\prime} & p+q
\end{array}\right),
\end{aligned}
$$

которые произведения матриц из $\mathscr{M}$ позволяют переводить в произведения матриц из множества $\mathscr{N}$ и наоборот.

ПримеР. Для дроби $5 / 7=[0 ; 1,2,1,1]$ множества $A_{1}, A_{2}$ и $A_{3}$ имеют вид

$$
A_{1}=\{(1,1,2,5),(1,3,1,2),(3,4,1,1)\}, \quad A_{2}=A_{3}=\{(1,3,1,2)\} .
$$

Элементы $A_{1}$ находятся во взаимно однозначном соответствии с разложениями матрицы $\left(\begin{array}{ll}3 & 5 \\ 4 & 7\end{array}\right)=\Psi(1,2,1,1)$ в произведение элементов $\mathscr{M}$ :

$$
\left(\begin{array}{ll}
3 & 5 \\
4 & 7
\end{array}\right)=\left(\begin{array}{ll}
0 & 1 \\
1 & 1
\end{array}\right)\left(\begin{array}{ll}
1 & 2 \\
3 & 5
\end{array}\right)=\left(\begin{array}{ll}
1 & 2 \\
1 & 3
\end{array}\right)\left(\begin{array}{ll}
1 & 1 \\
1 & 2
\end{array}\right)=\left(\begin{array}{ll}
2 & 3 \\
3 & 4
\end{array}\right)\left(\begin{array}{ll}
0 & 1 \\
1 & 1
\end{array}\right) .
$$

По разложению $5 / 7=\langle 0 ; 1 / 1,1 / 1,1 / 1,-1 / 3\rangle^{\star}$ находится множество

$$
B=\{(1,1,2,5),(1,2,3,2),(2,3,-1,3)\},
$$

элементам которого соответствуют разложения матрицы $\left(\begin{array}{l}2 \\ 3\end{array} 7\right)=\Phi(5 / 7)$ в произведение матриц из $\mathscr{N}$ :

$$
\left(\begin{array}{ll}
2 & 5 \\
3 & 7
\end{array}\right)=\left(\begin{array}{ll}
0 & 1 \\
1 & 1
\end{array}\right)\left(\begin{array}{ll}
1 & 2 \\
2 & 5
\end{array}\right)=\left(\begin{array}{ll}
1 & 1 \\
1 & 2
\end{array}\right)\left(\begin{array}{ll}
1 & 3 \\
1 & 2
\end{array}\right)=\left(\begin{array}{ll}
1 & 2 \\
2 & 3
\end{array}\right)\left(\begin{array}{cc}
0 & -1 \\
1 & 3
\end{array}\right) .
$$

При этом

$$
B_{1}=\{(1,1,2,5)\}, \quad B_{2}=\{(1,2,3,2)\}, \quad B_{3}=\{(2,3,-1,3)\} .
$$

Аналогично, по дроби $2 / 7=\langle 0 ; 1 / 3,1 / 1,1 / 1\rangle^{\star}$ строится множество

$$
\widetilde{B}=\{(1,3,1,2),(3,4,1,1)\},
$$

элементы которого соответствуют разложению матрицы $\left(\begin{array}{ll}1 & 2 \\ 3 & 7\end{array}\right)=\Phi(2 / 7)$ в произведение матриц из $\mathscr{N}$ :

$$
\left(\begin{array}{ll}
1 & 2 \\
3 & 7
\end{array}\right)=\left(\begin{array}{ll}
0 & 1 \\
1 & 3
\end{array}\right)\left(\begin{array}{ll}
1 & 1 \\
1 & 2
\end{array}\right)=\left(\begin{array}{ll}
1 & 1 \\
3 & 4
\end{array}\right)\left(\begin{array}{ll}
0 & 1 \\
1 & 1
\end{array}\right)
$$

При этом

$$
\widetilde{B}_{1}=\{(1,3,1,2),(3,4,1,1)\}, \quad \widetilde{B}_{2}=\widetilde{B}_{3}=\varnothing .
$$

Легко видеть, что $f_{i}\left(A_{i}\right)=B_{i} \cup \widetilde{B}_{i}, i=1,2,3$. 


\section{5. Основные результаты.}

Лемма 3. Пусть $b \geqslant 2,(a, b)=1,1 \leqslant a, a^{\star}<b, a a^{\star} \equiv 1(\bmod b)$. Тогдa

$$
h^{\star}\left(\frac{a}{b}\right)+h^{\star}\left(\frac{b-a}{b}\right)=h\left(\frac{a^{\star}}{b}\right)+h\left(\frac{b-a^{\star}}{b}\right) .
$$

ДокАЗАТЕЛЬСтво. Дополняя столбцы $\left(\begin{array}{l}a \\ b\end{array}\right),\left(\begin{array}{c}b-a \\ b\end{array}\right)$ до матриц из $\mathscr{N}$, при $\Delta(a / b)=1$ получим матрицы

$$
\left(\begin{array}{cc}
* & a \\
b-a^{\star} & b
\end{array}\right), \quad\left(\begin{array}{cc}
* & b-a \\
a^{\star} & b
\end{array}\right)
$$

а при $\Delta(a / b)=-1-$ матрицы

$$
\left(\begin{array}{cc}
* & a \\
a^{\star} & b
\end{array}\right), \quad\left(\begin{array}{cc}
* & b-a \\
b-a^{\star} & b
\end{array}\right) .
$$

В любом случае утверждение леммы вытекает из свойства $4^{\circ}$.

ДокАЗАТЕЛЬСтво теОРЕмЫ. Применяя леммы 2 и 3, находим

$$
\begin{aligned}
\sum_{a=1}^{b} h\left(\frac{a}{b}\right) & =\sum_{a=1}^{b} h\left(\frac{a^{\star}}{b}\right)=\sum_{a=1}^{b} h^{\star}\left(\frac{a}{b}\right) \\
& =\sum_{b / 2 \leqslant a \leqslant b}^{*}\left(h^{\star}\left(\frac{a}{b}\right)+h^{\star}\left(\frac{b-a}{b}\right)\right)=\sum_{b / 2 \leqslant a \leqslant b}^{*}\left(s_{\varphi}\left(\frac{a}{b}\right)+s_{\varphi-1}\left(\frac{a}{b}\right)\right) .
\end{aligned}
$$

Если $a / b=\left[0 ; 1, a_{1}, a_{2}, \ldots, a_{s}, 1\right]$, то $(b-a) / b=\left[0 ; a_{1}+1, a_{2}, \ldots, a_{s}, 1\right]$. Поэтому, по определению статистик Гаусса-Кузьмина

$s_{x}\left(\frac{a}{b}\right)-s_{x}\left(\frac{b-a}{b}\right)=\chi_{[0,\{x\}]}\left(\frac{a}{b}\right)+\chi_{[0, x]}\left(\frac{b-a}{b}\right)-\chi_{[0,\{x\}]}\left(\frac{b-a}{b}\right)-\chi_{[0, x]}\left(\frac{a}{b-a}\right)$,

где $\chi_{I}$ - характеристическая функция отрезка $I$. В частности,

$$
s_{\varphi}\left(\frac{a}{b}\right)=s_{\varphi}\left(\frac{b-a}{b}\right), \quad s_{\varphi-1}\left(\frac{a}{b}\right)=s_{\varphi-1}\left(\frac{b-a}{b}\right) .
$$

Значит,

$$
\sum_{a=1}^{b} h\left(\frac{a}{b}\right)=\frac{1}{2} \sum_{a=1}^{b} *\left(s_{\varphi}\left(\frac{a}{b}\right)+s_{\varphi-1}\left(\frac{a}{b}\right)\right),
$$

а следовательно, и

$$
\sum_{1 \leqslant a \leqslant b} h\left(\frac{a}{b}\right)=\frac{1}{2} \sum_{1 \leqslant a \leqslant b}\left(s_{\varphi}\left(\frac{a}{b}\right)+s_{\varphi-1}\left(\frac{a}{b}\right)\right) .
$$

Применяя равенства (8) и (9), приходим к утверждению теоремы. 


\section{СПИСОК ЦИТИРОВАННОЙ ЛИТЕРАТУРЫ}

[1] B. Vallée, "Dynamical analysis of a class of Euclidean algorithms", Theoret. Comput. Sci., 297:1-3 (2003), 447-486.

[2] H. Heilbronn, "On the average length of a class of finite continued fractions", Number Theory and Analysis, Papers in Honor of Edmund Landau, Plenum, New York, 1969, $87-96$.

[3] J. W. Porter, "On a theorem of Heilbronn", Mathematika, 22:1 (1975), 20-28.

[4] D. E. Knuth, "Evaluation of Porter's constant", Comput. Math. Appl., 2:2 (1976), 137-139.

[5] А.В. Устинов, "Асимптотическое поведение первого и второго моментов для числа шагов в алгоритме Евклида", Изв. РАН. Сер. матем., 72:5 (2008), 189-224.

[6] G. J. Rieger, "Über die mittlere Schrittanzahl bei Divisionsalgorithmen", Math. Nachr., 82:1 (1978), 157-180.

[7] G. J. Rieger, "Ein Heilbronn-Satz für Kettenbrüche mit ungeraden Teilnennern", Math. Nachr., 101:1 (1981), 295-307.

[8] V. Baladi, B. Vallée, "Euclidean algorithms are Gaussian", J. Number Theory, 110:2 (2005), 331-386.

[9] М. О. Авдеева, "О статистиках неполных частных конечных цепных дробей", Функи. анализ и его прил., 38:2 (2004), 1-11.

[10] Д.Э. Кнут, Искусство программирования. Т. 2: Получисленные алгоритмы, М., Вильямс, 2000.

[11] А.В. Устинов, "О числе решений сравнения $x y \equiv l(\bmod q)$ под графиком дважды непрерывно дифференцируемой функции", Алгебра и анализ, 20:5 (2008), 186-216.

[12] А. В. Устинов, "О среднем числе шагов в алгоритме Евклида с выбором минимального по модулю остатка", Матем. заметки, 85:1 (2009), 153-156.

\section{А. В. Устинов}

Хабаровское отделение

Института прикладной математики ДВО РАН

E-mail: ustinov@iam.khv.ru
Поступило

13.04 .2010 